\title{
The role of subgroup analyses: results from the NOVA trial
}

\author{
Massimo Di Maio $^{1}$, Alice Bergamini²
}

\begin{abstract}
The clinical efficacy of poly-adenosine diphosphate (ADP) ribose polymerase (PARP) inhibitors in BRCA-mutated ovarian cancer patients has been widely reported. However, novel challenges are currently aimed at broadening the benefit of PARP inhibitors to patients with wild-type BRCA and homologous recombination deficiency (HRD) and identifying a test able to select those patients who might benefit most from this therapy. The recently published ENGOT-OV16/NOVA trial reported an advantage in progression-free survival (PFS) for patients receiving the PARP1/2 inhibitor niraparib, as maintenance treatment for platinum-sensitive ovarian cancer compared to placebo, regardless of their germline BRCA or HRD status. This suggests that niraparib could potentially represent a valuable maintenance option for virtually all patients with platinum-sensitive relapsed ovarian cancer and that the HRD assay used in this trial is not able to reliably identify those patients who benefit the most from niraparib maintenance. These provocative considerations are the result of subgroup analyses that should be interpreted with caution but are useful to show the consistency of the effect of niraparib across the whole population.
\end{abstract}

Key words: PARP inhibitors, niraparib, ovarian cancer, HRD, BRCA, subgroup analyses

\section{Introduction}

The clinical efficacy of poly-adenosine diphosphate (ADP) ribose polymerase (PARP) inhibitors in BRCAmutated ovarian cancer patients has been widely documented [1-4]. However, novel challenges are currently aimed at (1) broadening the benefit of PARP inhibitors to patients with wild-type BRCA and homologous recombination deficiency (HRD) and (2) identifying a test able to select those patients who might benefit the most from this therapy.

The recently published European Network of Gynaecological Oncological Trial Groups (ENGOT) ENGOTOV16/NOVA trial, a randomized, double-blind, phase 3 trial assessing the efficacy and safety of niraparib, a

'Department of Oncology, University of Turin, Mauriziano Hospital, Turin, Italy.

${ }^{2}$ Department of Obstetrics and Gynecology, San Raffaele

Hospital, Milan, Italy.

Correspondence to:

Alice Bergamini,

Dipartimento di Ostetricia e Ginecologia, Ospedale San Raffaele,

Via Olgettina 60, 20132 Milano, Italy.

Phone: +3902 26435595 - Fax: +39 0226432759

E-mail: bergamini.alice@hsr.it

CANCER BREAKING NEWS 2017;5(1):40-42

DOI: 10.19156/cbn.2017.0039
PARP1/2 inhibitor versus placebo, has tried to focus on these issues in the setting of maintenance therapy for patients with platinum-sensitive, recurrent ovarian cancer [5].

The primary endpoint of the study was progression-free survival (PFS); secondary endpoints included patient reported outcomes, chemotherapy-free interval, overall survival (OS) and intermediate outcome measures such as time to first subsequent therapy (TFST), progressionfree survival 2 (considering the progression during next anticancer therapy), and time to second subsequent therapy (TSST).

\section{Methodological comment}

In evaluating the efficacy of niraparib, patients were categorized as germline BRCA mutation carriers (gBRCA) and non-carriers (non-gBRCA cohort).

The efficacy analysis was performed in three predefined primary efficacy populations: the gBRCA cohort, the HRD-positive subgroup of the non-gBRCA cohort and the overall non-gBRCA cohort. In detail, statistical analysis was planned according to a predefined hierarchical-testing procedure for the non-gBRCA cohort: comparison was first performed in patients with HRD-positive tumors, and only in case of a significant result the test was extended to the overall non-gBRCA cohort. 
The duration of PFS was significantly longer in the niraparib group, compared to placebo, in all the three cohorts of patients (21.0 vs 5.5 months, hazard ratio [HR] 0.27 for the gBRCA group; 12.9 vs 3.8 months, HR 0.38 for the HRD-positive non-gBRCA group and 9.3 vs 3.9 months, HR 0.45 for non-gBRCA patients, respectively).

A test of interaction between treatment and subgroup factors was performed to ensure the consistency of the treatment effect. Of interest, a prespecified exploratory subgroup analysis of PFS was conducted within three populations of non-gBRCA patients, to assess the role of a potential biomarker in driving the effect of niraparib maintenance therapy in the gBRCA-negative cohort. The three populations comprised patients with (1) HRD-positive plus somatic BRCA mutation, (2) HRD-positive plus wild-type BRCA and (3) HRD-negative status, respectively. A benefit from niraparib treatment was detected in all three subgroups, even if with different magnitudes (20.9 vs 11.0, HR 0.27; 9.3 vs 3.7, HR 0.38 and 6.9 vs 3.8 months, HR 0.58 , respectively).

This consideration has two direct implications. Firstly, niraparib could potentially represent a valuable maintenance option for virtually all patients with platinumsensitive relapsed ovarian cancer. Secondly, although the absolute outcome is quite different among the molecular subgroups, a significant benefit was shown with niraparib within each subgroup, implying that the HRD assay used in this trial is not able to significantly identify those patients who benefit the most from niraparib maintenance.

Thus, the ideal biomarker to potentially broaden the benefit from PARP inhibitors to HRD patients is currently still lacking. Another HRD assay based on genomic loss of heterozygosity ( $\mathrm{LOH}$ ) estimation, being assessed in the recently published ARIEL2 study [6], will hopefully identify patients most likely to respond to rucaparib treatment in the ongoing ARIEL3 trial (NCT01968213).

However, these provocative considerations of the NOVA trial are the result of a subgroup analysis, even if prespecified. The role, limitations and challenges posed by subgroup analyses in randomized clinical trials have been highlighted by several authors and should always be taken into account while critically evaluating the results [7-9].

The introduction of subgroup analyses is generally the result of both regulatory agencies and the sponsor's interests to identify patient subgroups who might benefit the most from trial intervention.

When a positive result has been obtained in the whole study population, as in the case of the NOVA trial, sub- group analyses may be useful to show the consistency or heterogeneity of the treatment effect. In general, these analyses should better be confined to the primary outcome and should involve only a few prespecified subgroups based on a biologically realistic hypothesis, as in the case of the NOVA trial. Although conclusions deriving from these analyses should be interpreted with caution, the evaluation of the efficacy of the experimental treatment in important subpopulations can be scientifically and clinically justified, also from the point of view of regulatory agencies. In fact, as effectively discussed in the "Guideline on the Investigation of Subgroups in Confirmatory Clinical Trials" published by the European Medicines Agency in 2014, verifying that the conclusions of efficacy and safety can be applied across the different subgroups of the population enrolled into a clinical trial is relevant for the subsequent use of the drug in clinical practice [10].

Of course, subgroup analyses are potentially impaired by the multiplicity of statistical tests and a higher risk of false positive results, and this would be particularly dangerous in the case of a negative result in the whole study population, together with a positive result in one or more subgroups. However, this was not the case of the NOVA trial, given that the results of subgroup analyses are consistent with those obtained in the overall population. Furthermore, since the reduced statistical power in the subgroups with an increased rate of false negatives can be an issue, if the subgroup analyses of the NOVA trial had shown an absence of efficacy for niraparib in a specific subpopulation, it would have been difficult to interpret this finding as a false negative result or a real absence of efficacy.

As a general rule, in fact, when subgroup analyses suggest heterogeneity of treatment effect, they have to be considered exploratory; useful to generate hypotheses to be confirmed in further prospective trials.

\section{Acknowledgments}

The authors thank Ray Hill, an independent medical writer, who provided native English editing and journal styling on behalf of HPS.

This editorial assistance was funded by PharmaMar, Spain.

\section{Conflicts of Interest}

The Authors declare there are no conflicts of interest in relation to this article. 


\section{References}

1. Ledermann J, Harter P, Gourley C et al. Olaparib maintenance therapy in platinum-sensitive relapsed ovarian cancer. N Engl J Med 2012;366(15):1382-92.

2. Gelmon KA, Tischkowitz M, Mackay H et al. Olaparib in patients with recurrent high-grade serous or poorly differentiated ovarian carcinoma or triple-negative breast cancer: a phase 2, multicentre, open-label, non-randomised study. Lancet Oncol 2011;12(9):852-61.

3. Sandhu SK, Schelman WR, Wilding G et al. The poly(ADPribose) polymerase inhibitor niraparib (MK4827) in BRCA mutation carriers and patients with sporadic cancer: a phase 1 dose-escalation trial. Lancet Oncol 2013;14(9):882-92.

4. Miller RE, Ledermann JA. The status of poly(adenosine diphosphate-ribose) polymerase (PARP) inhibitors in ovarian cancer, part 2: extending the scope beyond olaparib and BRCA1/2 mutations. Clin Adv Hematol Oncol 2016;14(9):704-11.

5. Mirza MR, Monk BJ, Herrstedt J et al. Niraparib mainte- nance therapy in platinum-sensitive, recurrent ovarian cancer. N Engl J Med 2016;375(22):2154-64.

6. Swisher EM, Lin KK, Oza AM et al. Rucaparib in relapsed, platinum-sensitive high-grade ovarian carcinoma (ARIEL2 Part 1): an international, multicentre, open-label, phase 2 trial. Lancet Oncol 2017;18(1):75-87.

7. Di Maio M, Perrone F. Subgroup analysis: Refining a positive result or trying to rescue a negative one? J Clin Oncol 2015;33(36):4310.

8. Wang R, Lagakos SW, Ware JH et al. Statistics in medicine-reporting of subgroup analyses in clinical trials. N Engl J Med 2007;357(21):2189-94.

9. Cook DI, Gebski VJ, Keech AC. Subgroup analysis in clinical trials. Med J Aust 2004;180(6):289-91.

10. European Medicines Agency. Guideline on the investigation of subgroups in confirmatory clinical trials, 2014. Available from: http://www.ema.europa.eu/docs/en_GB/document library/Scientific_guideline/2014/02/WC500160523.pdf. 